\title{
Sensor Deployment and Mobile Target Detection in Wireless Sensor Networks
}

\author{
S.Sujana \\ Associate Professor \\ Vardhaman College of \\ engineering \\ Hyderabad, India
}

\author{
A.Bhargavsagar \\ Student of M .Tech \\ Vardhaman College of \\ engineering \\ Hyderabad, India
}

\begin{abstract}
WSNs are generally composed of small-sized battery-operated devices with embedded sensing and wireless communication capabilities. We propose an optimization framework for the problem of positioning sensors where the sensing quality depends on the distance from the sensor node. In our approach, we consider two main objectives: minimizing the number of nodes deployed and maximizing the exposure path. In our proposed model, we implemented normal wifi modem as the sensors. So we can reduce the unnecessary separate sensor deployment.
\end{abstract}

\section{Keywords}

WSN, Wifi, Mobile target, Exposure path.

\section{INTRODUCTION}

A wireless sensor network consists of sensor nodes capable of collecting information from the environment and communicating with each other via wireless transmitter and receivers. The collected data will be delivered to one or more sinks, generally through multi-hop communication. A wireless sensor network consists of spatially distributed autonomous sensors to monitor physical or environmental status, such as sound, heat, pressure, etc. and to cooperatively pass their data through the network to a main location. The more modern networks are duplex, also enabling control of sensor process. Development of wireless sensor networks was motivated by military applications such as battlefield monitoring; today such networks are used in many industrial and consumer applications, such as industrial process checking, machine health checking, and so on.

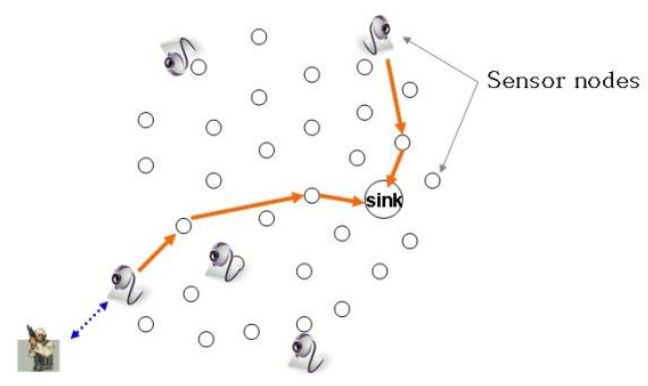

\section{RELATED WORK}

In [1] this article, researcher surveyed recent contributions addressing energy-efficient coverage problems in the context of static WSN. Sensor coverage is an important element for QoS in applications with WASNs. Sensor coverage is still limited to theoretical study. In [2] this paper, authors were interested in measuring the quality of barrier coverage. The method is equivalent to measuring its quality as either 1 or 0 . The measured quality is short of a desired value, author further identified all local regions that need to be repaired.
This mechanism is efficient is to monitor network properties of sensors. If one of them is not repaired then the resulting network will still be short of quality. In [3] this paper, author characterizes three fundamental coverage measures of largescale sensor networks: area and node coverage, and detestability and the asymptotic behavior of the coverage measures for a variety of sensor network scenarios. This mechanism is efficient is to monitor network properties of sensors. Protocol performance of sensor networks is not considered. In [4] this paper author has defined a shortest-path network-interdiction problem, and Maximizing the Shortest Path on a directed network. This mechanism overcomes delay. But the algorithms are ineffective when delays are large. In [5] this paper, all important aspects of sensor network is described including sensor nodes, network architecture, protocols and protocol stack, way of energy efficiency etc. Media access control, security and privacy and efficient routing protocols are not discussed in this paper.

\section{SYSTEM IMPLEMENTATION}

\subsection{Existing System}

Previous work on mobile target detection using the concept of exposure focuses on assessing the performance of randomly deployed WSNs. And also previous work on this topic relies on the general 1-0 coverage model based on the sensing range.

\subsubsection{Problem Statement}

In the classic coverage model, the entire area must be monitored to detect targets. Here, instead the goal is to detect a target that crosses a protection border, moving from an external area to a sensible area. Therefore, the objective is not to detect an intruder at any point in the area, but to detect the crossing attempt.

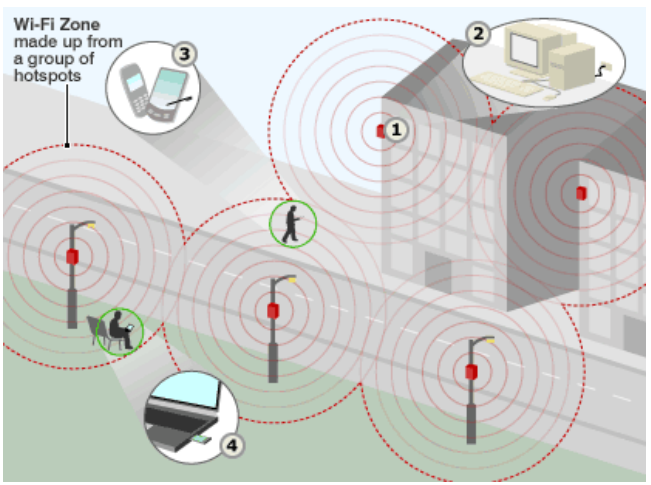




\subsection{Proposed System}

We propose an optimization framework for selecting the positions of wireless sensors to detect mobile targets traversing a given area. In our approach, we consider two main objectives: minimizing the number of nodes deployed and maximizing the exposure of the least-exposed path.

\subsubsection{Advantages}

Unlike in previous work based on coverage quality, the capability of the network to detect a mobile object moving along a given path depends on the distance from sensors and is measured by the path exposure.
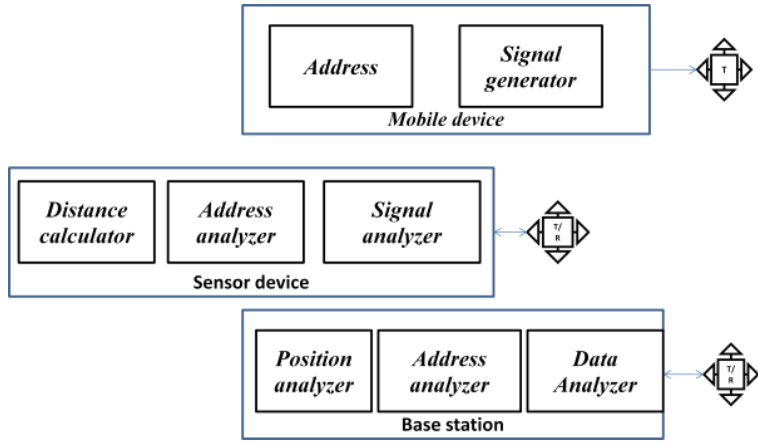

Fig C. Mobile Target detection model

\section{System Description}

To make efficient system, proposed model is divided into four modules.

\subsection{Deploying the Sensor Nodes}

The design of a WSN for movable target detection requires a completely different deployment approach. We are implementing two methods.

The first method is Sensors must be positioned in order to maximize the exposure of the least-exposed path, subject to a budget on the installation cost (number of sensors). The second method is Sensors have to be placed so as to minimize the installation cost, provided that the exposure of the leastexposed path is above a given threshold.

\subsection{Creating the Mobile Target}

We need to create mobile device, it's should be satisfy following contains

Able to move from one position to another place

It has to generate beacon message in short interval with following metrics. Timestamp, Mobile device address, Broadcast ID, Sequence number.

\subsection{Creating Sensor Nodes}

We need to create sensor device, it's should be satisfy following contains

We need to create sensor device, it's should be satisfy following contains.

Able to receive beacon message from mobile node and also can analyze the following metrics.

Time stamp, Mobile device address, Broadcast ID, Sequence number

And it is able to calculate distance $\mathrm{b} / \mathrm{w}$ mobile target and sensor.

\subsection{Creating Base Station Node}

We need to create base station device, it's should be satisfy following contains

Able to receive analyzed data from sensor device.

Able to find the position by using following conations.

Time comparison, Find sensor address \& position, Distance comparison, calculate the mobile node position.

\section{TRACING MOBILE TARGET}

In our project, we made the mobile target can produce the beacon signal in periodic interval. And sensor node can able to sense the signals as shown in Fig.1a and it can make the distance calculation.

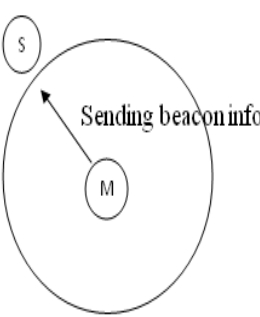

Fig. 1a Beacon sharing

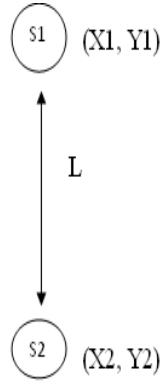

Fig.1b Distance $b / w$ sensors
According to our theory, mobile target signal propagation speed is $\mathrm{S} \mathrm{m} / \mathrm{s}$. And distance is calculation is

$$
\begin{aligned}
D(i) & =\frac{\text { time delay }(i)}{\text { speed of the signal }} \\
\mathrm{i} & =\text { node number }
\end{aligned}
$$

We deployed the sensor in some fixed position as shown in Fig.1b and so each and every sensor node position is known by the base station.

So base station can calculate distance b/w sensor by already known

$$
L=\sqrt{\left(X_{2}-X_{1}\right)^{2}+\left(Y_{2}-Y_{1}\right)^{2}}
$$

Where $\mathrm{L}$ is distance $\mathrm{b} / \mathrm{w}$ sensors, $\mathrm{X} 1, \mathrm{X} 2, \mathrm{Y} 1, \mathrm{Y} 2$ are position of sensor (check fig).

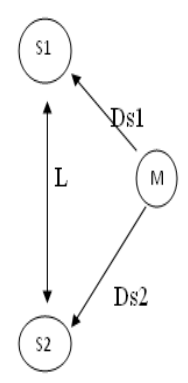

Fig.2a distance $\mathrm{b} / \mathrm{w}$ sensors and MT

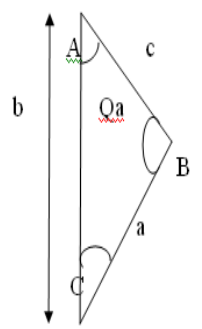

Fig.2b equivalent diagram 
Fig.2a shows the real model of sensors and fig. $2 \mathrm{~b}$ shows the equivalent diagram of fig. In that picture $\mathrm{A}, \mathrm{C}$ and $\mathrm{B}$ are sensors and mobile target respectively. And a, b, c are distance.

To find exact position of mobile target we need to calculate angle sensor axis Qa or Qb or Qc (from fig.2b).

To calculate we can use following formula.

$$
\cos Q_{a}=\frac{\left(b^{2}+c^{2}-a^{2}\right)}{2 a b}
$$

Then modified diagram of fig. $2 \mathrm{~b}$ is shown below in fig. $3 \mathrm{a}$

Fig. $3 \mathrm{~b}$ is converted diagram of Fig.3a. To find exact position of mobile target we should calculate the distance as X and Y given in fig. $3 b$
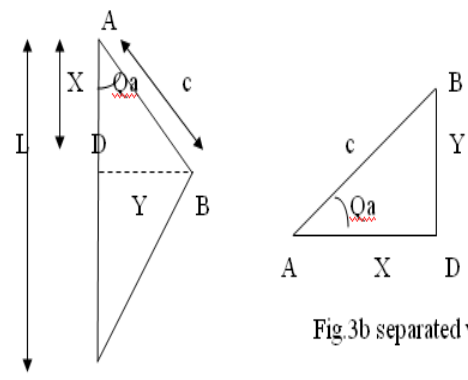

Fig. $3 b$ separated value

Fig.3a separation

$$
\begin{aligned}
& \cos Q_{a}=\frac{X}{c} \\
& \sin Q_{a}=\frac{Y}{c}
\end{aligned}
$$

From above equation we can calculate exact position is possible.

\section{RESULT}

We have analyzed the network with optimal sensor placement system. To make simulation study we used Ns2. In this analyze, 14 nodes placed. There are 1 mobile target node and one base station node and remaining all are wifi base sensor devices.

We got the result with animation and recorded file. From animation we can check mobile target position. And from recorded data, we can check performance our algorithm.

Table. 1 shows the result of distance $\mathrm{b} / \mathrm{w}$ sensor and mobile target. And Table. 2 shows the result of exact position of the mobile target node.
Table: 1 coverage sensors details

\begin{tabular}{|l|l|l|}
\hline \multirow{2}{*}{$\begin{array}{l}\text { Seq. } \\
\text { no }\end{array}$} & $\begin{array}{l}\text { Coverage } \\
\text { sensor } \\
\text { name }\end{array}$ & $\begin{array}{l}\text { Distance b/w MT } \\
\text { and Sensor(m) }\end{array}$ \\
\hline \multirow{2}{*}{1} & 0 & 113.0 \\
\cline { 2 - 3 } & 6 & 112.0 \\
\hline \multirow{5}{*}{2} & 0 & 150.89 \\
\cline { 2 - 3 } & 1 & 123.56 \\
\cline { 2 - 3 } & 6 & 150 \\
\cline { 2 - 3 } & 7 & 122.65 \\
\hline \multirow{5}{*}{3} & 1 & 123.56 \\
\cline { 2 - 3 } & 2 & 150.76 \\
\cline { 2 - 3 } & 7 & 122.56 \\
\cline { 2 - 3 } & 8 & 150 \\
\hline \multirow{5}{*}{4} & 2 & 113 \\
\cline { 2 - 3 } & 8 & 112 \\
\hline 5 & 2 & 150.89 \\
\cline { 2 - 3 } & 3 & 123.56 \\
\cline { 2 - 3 } & 9 & 122.65 \\
\cline { 2 - 3 } & 10 & 150.146 \\
\hline 6 & 3 & 123.89 \\
\hline \multirow{5}{*}{6} & 4 & 150.89 \\
\hline & 9 & 122.65 \\
\hline & 10 & \\
\hline
\end{tabular}

Table: 2. exact positions of the node

\begin{tabular}{|l|l|l|}
\hline Seq. no & X-pos(m) & Y-pos(m) \\
\hline 1 & 100 & 213 \\
\hline 2 & 300.0 & 213 \\
\hline 3 & 500.0 & 213 \\
\hline 4 & 100 & 213 \\
\hline 5 & 300.0 & 213 \\
\hline 6 & 500.0 & 213 \\
\hline
\end{tabular}

In Nam window result, there are the sensors placed in optimal position to track mobile target. Sensed beacon information's are converted as distance information then sensor forwarding distance information to base station (shown in fig D and fig E)

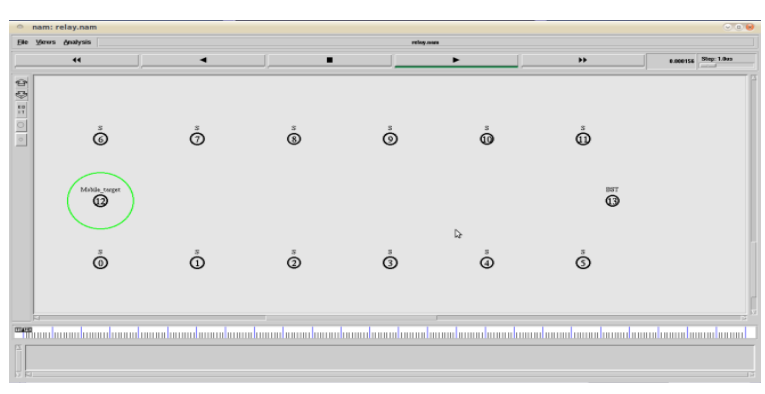

Fig D. Beacon sharing from mobile target 


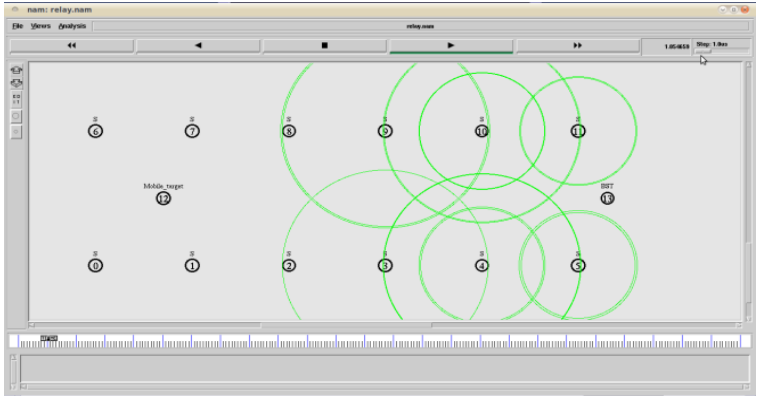

Fig E. Forwarding distance info to BS

\section{CONCLUSION}

We have proposed an optimization framework to place the sensors of a WSN in order to achieve high detection quality along path. Unlike in previous work based on coverage quality, the capability of the network to detect a mobile object moving along a given path depends on the distance from sensors and is measured by the path exposure. We achieved to target detection with maximum quality.

\section{ACKNOWLEDGMENTS}

The authors wish to thank the efforts of CE3iT, a research unit from Pantech group, India, who provided the valuable assistance to the undertaking of the research summarized here.

\section{REFERENCES}

[1] Design of Wireless Sensor Networks for Mobile Target Detection Edoardo Amaldi, Antonio Capone, Senior
Member, IEEE, Matteo Cesana, Member, IEEE, and Ilario Filippini, Member, IEEE., 2012

[2] Energy-Efficient Coverage Problems in Wireless Ad Hoc Sensor Networks. Mihaela Cardei and Jie Wu., 2010

[3] Measuring and Guaranteeing Quality of BarrierCoverage in Wireless Sensor Networks. Ai Chen,Ten H. Lai,Dong Xuan., May 30, 2008.

[4] A Study of the Coverage of Large-scale Sensor Networks. Benyuan Liu Don Towsley. 25-27 Oct. 2004.

[5] Shortest-Path Network, Eitan Israeli, R. Kevin Wood.2002.

[6] A Survey on Sensor Network, Kazi Chandrima Rahman.2002

[7] The Coverage Problem in Three-Dimensional Wireless Sensor Network, Chi-Fu Huang, Yu-Chee Tseng, and LiChu Lo. 2010

[8] Maintaining Sensing Coverage and Connectivity in Large Sensor Networks, Honghai Zhang and Jennifer C. Hou. 2005

[9] Stochastic Coverage in Heterogeneous Sensor Networks, Honghai Zhang and Jennifer C. Hou.2009 